\title{
Canine leishmaniasis: the key points for qPCR result interpretation
}

\author{
Verónica Martínez ${ }^{1 *}$, Javier Quilez ${ }^{1}$, Armand Sanchez $^{2}$, Xavier Roura $^{3}$, Olga Francino ${ }^{2}$ and Laura Altet ${ }^{2}$
}

\begin{abstract}
Background: Diagnosis and follow up of CanL is difficult since the range of clinical signs is varied and seroprevalence is high in endemic areas. The aims of this study were: i) demonstrate the advantages of Leishmania qPCR to diagnose and control CanL and highlight its prognostic value and ii) propose guidelines for tissue selection and infection monitoring.

Findings: This study included 710 dogs living in an endemic area of leishmaniasis. Forty percent (285/710) exhibited clinical signs consistent with CanL. Infection was detected in 36.3\% (258/710) of the dogs of which 4.5\% (32/710) were detected by qPCR, 16.2\% (115/710) detected by ELISA and 15.6\% (111/710) tested positive for both tests. Only $17.9 \%(127 / 710)$ of the dogs were classified sick (affected) with CanL.

All symptomatic dogs with medium or high ELISA titers were GPCR-positive in blood samples. All dogs with inconclusive or low ELISA results with high or medium QPCR parasitemia values developed the disease. Seventy one percent of asymptomatic ELISA-positive dogs confirmed by qPCR (medium to high parasitemia) developed the disease. Bone marrow or lymph node aspirate should be selected to ensure the absence of the parasite in asymptomatic dogs: 100-1,000 parasites/ml in bone marrow are detectable in blood, whereas lower parasite loads are usually negative. Almost 10\% of negative samples in blood were positive in conjunctival swabs.

Conclusions: Because qPCR allows parasite quantification, it is an effective tool to confirm a diagnosis of CanL in (i) cases of inconclusive ELISA results, (ii) when the dog has not yet seroconverted, or (iii) for treatment monitoring.
\end{abstract}

\section{Findings}

Leishmaniasis is one of the main zoonosis worldwide and in some countries it is a reason of concern for public health. Canine leishmaniasis (CanL) is of great importance in veterinary medicine since dogs are believed to be the main reservoir of this parasite for humans [1]. It is endemic along the Mediterranean basin, parts of east Africa, India, Central and South America and the incidence of infection is currently spreading to non endemic areas towards the north of Europe [2] and recently emerging in North America [3]. In addition, other species have come to be infected, such as cats [4], and horses [5]. Wild canids are competent reservoirs of Leishmania [6], increasing the risks for humans to acquire the disease in endemic areas. Therefore, there has been a great interest in the development of new diagnostic tests.

\footnotetext{
* Correspondence: veronicalucia.martinez@uab.cat

'Departament de Ciència Animal i dels Aliments, Facultat de Veterinària,

Universitat Autònoma de Barcelona (UAB), 08193 Bellaterra, Barcelona, Spain Full list of author information is available at the end of the article
}

Diagnosis of CanL is fairly difficult, since dogs manifest a very varied range of clinical signs. In CanL, infection does not equal to having the clinical disease due to a high prevalence of subclinical infections $[7,8]$. Moreover, it is specially challenging in endemic areas where seroprevalence rates are high [7]. Epidemiological studies in endemic zones of CanL, by means of molecular techniques, have shown that the prevalence of infection in the canine population by Leishmania is considerably higher than seroprevalence [8]. There are several diagnostic tests for CanL, but the correct interpretations of these are of great importance to make an accurate diagnosis of the disease [9]. Therefore, the aims of this study were: i) to demonstrate the advantages of the quantitative PCR of Leishmania (qPCR) to diagnose and control the disease and highlight its prognostic value and ii) propose a guideline for the tissue of choice to be analyzed in each case, as well as a guideline for monitoring the disease.

The study included 710 dogs from the LUPA Project (7 PM; subWP canine leishmaniasis). The LUPA project 
http://www.eurolupa.org/ is a European initiative to study common complex human diseases using the dog as animal model. The UAB leads the subworkpackage focusing on canine leishmaniasis. All samples had informed owner consent. Data from medical history including physical exploration, clinical biochemistry and complete blood count were collected for most of the cases. Both anti-Leishmania ELISA and qPCR were performed on serum and blood samples, respectively. Serologies were performed at UNIVET $^{\circledR}$ using serum samples that were tested using INGEZIM Leishmania, an enzyme-linked immunoassay (ELISA) provided by Ingenasa (Madrid, Spain), with some modifications. Presence of antibodies against Leishmania was determined using anti-dog IgG as conjugate, following the recommendations of the manufacturer as described elsewhere [10]. qPCR was performed at the Servei Veterinari de Genètica Molecular of Universitat Autònoma de Barcelona as described by Francino et al. [11]. Table 1 offers the cutoff values for both ELISA and qPCR tests.

Dogs were monitorized from February 2007 until March 2010 and, according to the recorded data mentioned above, they were classified as affected or unaffected by the disease.

In 112 out of 710 dogs qPCR was additionally performed in at least one alternative tissue to detect infection of Leishmania (i.e. bone marrow, lymph node aspirate, biopsy and lesional or conjunctival swabs), providing useful information to support the tissue of choice for diagnosing CanL.

All data was compiled with Excel (Microsoft). The difference between groups was tested for significance by Chisquared analysis. A p value $<0.05$ was considered as statistically significant. For the observed prevalence, 95\% confidence interval (CI) was calculated. Differences between proportions were calculated using Chi-squared Yates correction. Test characteristics such as sensitivity, disease prevalence as well as positive predictive power, were analyzed from a $2 \times 2$ table using ROC curve analysis. All data was analyzed using MedCalc Software [12,13].

\section{qPCR prognostic value}

Forty percent (285/710) of the dogs exhibited clinical signs consistent with CanL. Overall detection of the parasite by
ELISA and/or qPCR was found in 36.3\% (258/710) of the samples, but only $17.9 \%(127 / 710)$ of the dogs were classified as affected of the disease. These results differ from higher values of prevalence showed in other studies where more tissues were analyzed in each dog [7], but reinforce the statement that further specific diagnostic tests such as serology and qPCR should be performed in order to reach a correct diagnosis. Treatment of dogs just based on few compatible clinical signs is not recommended, especially in endemic areas.

In endemic areas, a large part of the canine population is exposed to the parasite (positive serological results are expected) compared with the smaller proportion of dogs that eventually develop disease [7]. These results are also confirmed in our study; in which seroprevalence was of $31.8 \%(226 / 710)$ but only $50 \%$ of these dogs $(113 / 226)$ were classified as affected of CanL. Serology titers remain high for a longer period of time before antibodies levels decrease [14]. Moreover, false positive results in serology tests can occur due to cross reactivity with other pathogens [8]. On the other hand, dogs remain seronegative during variable time periods after the infection with Leishmania [15]. Intervals for seroconversion on naturally infected dogs can take from 1 to 22 months (median 5 months), and from 1 to 6 months (median, 3 months) for experimentally infected dogs [16]. Therefore, serology alone has a limited predictive value because the results may be affected by persistent antibodies or inmunosupression. This statement correlates with our results because we found that $84.3 \%(97 / 115)$ of the dogs, who had positive serologies and negative qPCRs, were classified as unaffected of CanL. Detection and accurate parasite quantification of the qPCR is an effective help in the CanL diagnosis, mostly in cases of uncertain serology results or when the dog has still not seroconverted. Prevalence according to qPCR was lower than seroprevalence in our study $(20.1 \%(143 / 710)$ but the majority of the dogs were classified as affected of the disease $[67.8 \%$ $(97 / 143)]$. In conclusion, qPCR has a higher positive predictive value $(67.8 \%$ vs. $50 \%$; p value: 0.001$)$ that will give us confidence when looking at test results.

All the dogs analyzed with clinical signs and medium or high positive ELISA titers were qPCR positive in blood samples, except those that received previous

Table 1 Categories for cutoff values of qPCR and ELISA

\begin{tabular}{|c|c|c|c|c|}
\hline qPCR result & Parasites $/ \mathrm{ml}$ of blood & Parasites/ml of bone marrow & ELISA result & Titer (\%) \\
\hline Negative & 0 & 0 & Negative & $<20$ \\
\hline Low positive & $0-10$ & $0-100$ & Uncertain* & $20-35$ \\
\hline Medium positive & $10-100$ & $100-1,000$ & Low positive & $35-80$ \\
\hline High positive & $100-1,000$ & $1,000-10,000$ & Medium positive & $80-150$ \\
\hline Very high positive & $>1,000$ & $>10,000$ & High positive & $>150$ \\
\hline
\end{tabular}

* This category was incorporated for the purpose of this study based on previous data (data not shown). 
treatment or only manifested dermatological signs, in which the tissue of choice should be a swab of the dermatological lesion. In dogs with clinical signs, but low or uncertain ELISA titers, the qPCR has a significant prognostic value. In our study, $100 \%$ of symptomatic dogs with inconclusive ELISA results (uncertain or low positive), but with patent parasite detection (high or medium parasitemia measured with qPCR) develop the disease (Figure 1).

In dogs without clinical signs, the qPCR also has an important prognostic value since $71.4 \%$ of asymptomatic ELISA-positive dogs confirmed by qPCR with medium to high parasitemia values will end up with patent leishmaniasis.

As a conclusion, dogs whose parasitemia range from medium to high or very high positive are sick or eventually will become sick with CanL.

\section{The tissue of choice}

Due to the varied tropism that the parasite exhibits regarding different tissues, the quantity of the parasite is different and oscillates among them [7]. It is important that the clinician chooses the most informative one in each case since qPCR can be performed on different tissues such as blood, bone marrow, lymph node, body fluids, histopathology samples or conjunctival swabs.

To ensure the absence of the parasite in dogs without clinical signs, the sample of choice is bone marrow or lymph node aspirate since it is been proved to be the most sensitive ones [17]. Moreover, blood is a valid tissue to perform routine qPCR analysis of CanL to evaluate response to the treatment. In our study, we found that in few samples in which both blood and bone marrow were analyzed, $77.8 \%(7 / 9)$ of the blood samples detected Leishmania while $88.9 \%$ (8/9) of the bone marrow did. The difference corresponds to two dogs that were asymptomatic and had very low titers in bone marrow ( 2 parasites $/ \mathrm{ml}$ ) and negative titers in peripheral blood. Parasite loads equal or greater than 100-1,000 parasites/ml in bone marrow are detectable in blood, whereas lower parasite loads (1-100 parasites/ml in bone marrow) are usually negative in blood, since there is a correlation between both tissues (bone marrow usually being higher than blood). In this, way blood is a valid tissue for monitoring treatment efficacy despite that the first qPCR diagnosis has been performed in bone marrow.

Almost $10 \%(8 / 83)$ of samples which were negative in blood were positive in conjunctival swab. This result correlates with other study that reported that $83 \%$ of the dogs experimentally infected with $L$. infantum were already positive by PCR of conjunctival swabs at 6 weeks after infection, whereas only $17 \%$ of the buffy coat samples obtained at the same time were found to be positive [15]. Prevalence values increase if the dog shows ocular signs such as conjunctivitis, uveitis, blepharitis or periocular alopecia. The prevalence of ocular lesions in dogs with leishmaniasis range from $16 \%$ to $80 \%$ according to various studies $[18,19]$. Therefore, the tissue of choice in cases of ocular signs is conjunctival swab. It could also be used for early diagnosis since sensitivity is superior to serologic testing or parasite culture [15] and samples are obtained in a less invasive manner.

In the same way, lesional swab is recommended when only dermatological lesions are present. Other studies report that $65 \%$ of the dogs were found to be PCR positive for skin lesions compatible with Leishmania [15]. All together, we suggest guidelines for the tissue of choice for qPCR given prior clinical signs and ELISA results (Figure 2).

\section{Monitoring CanL}

In cases of positive qPCR it is recommended to monitor the parasite load one month after treatment to evaluate its response. If parasite titer decreases, treatment is effective and a new qPCR control is just recommended at the end of treatment (6-12 months). None of the current anti-leishmanial drugs that are being used have proved to induce parasitological remission in dogs since

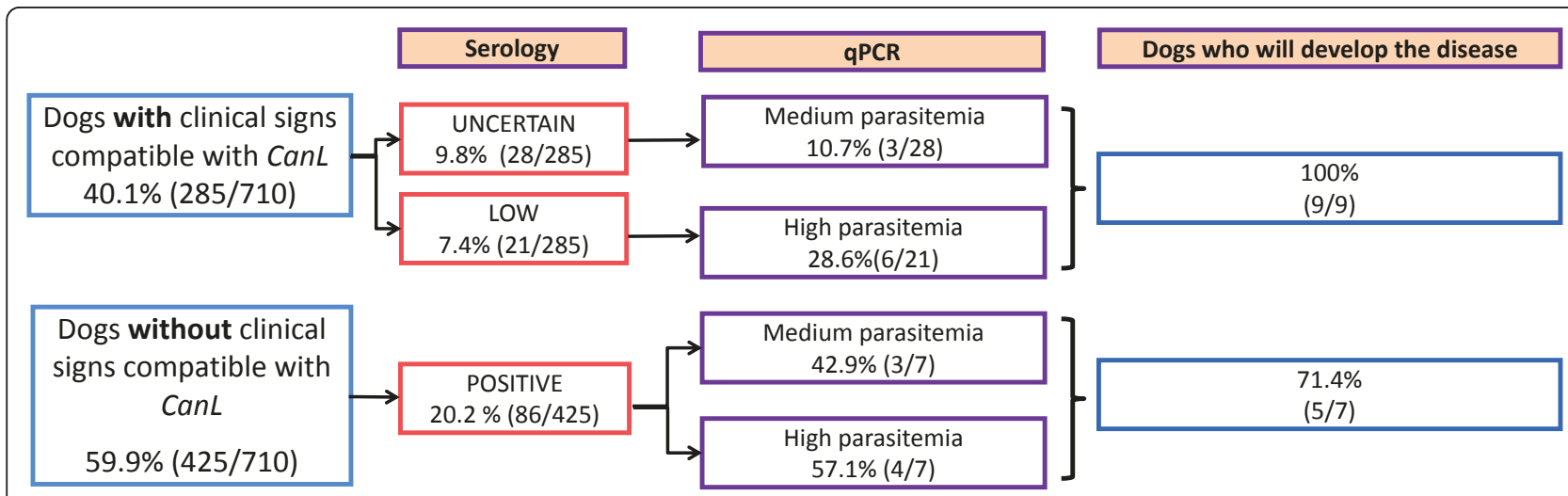

Figure 1 Prognostic value of the qPCR in uncertain cases of canine leishmaniasis 


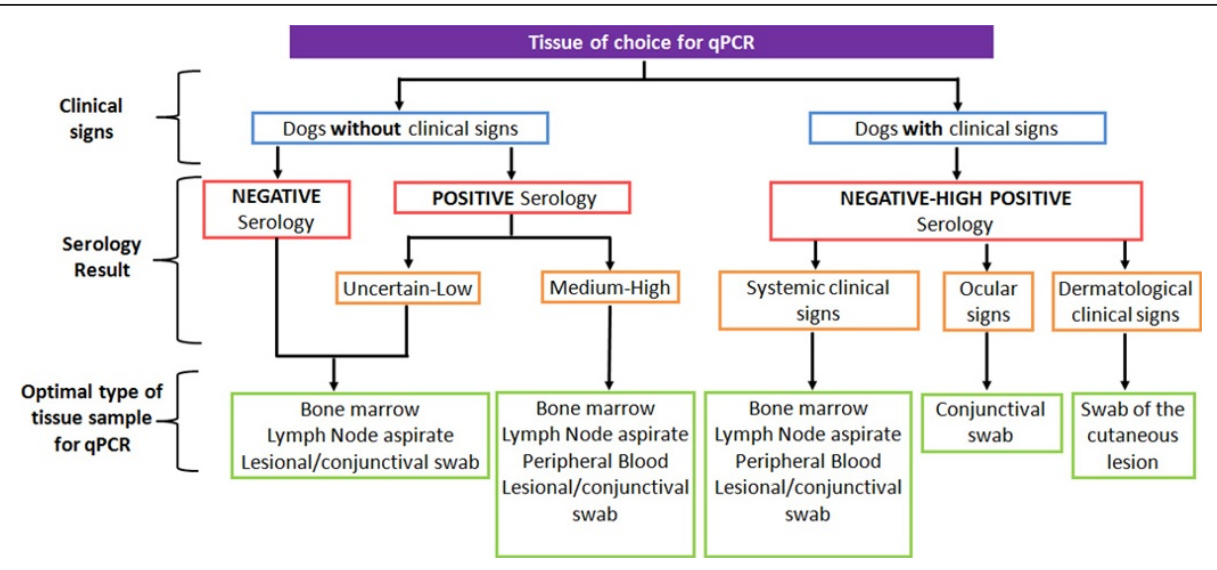

Figure 2 Tissue of choice for $\mathrm{qPCR}$.

a small parasite load usually remains in the majority of cases $[20,21]$. If the parasite load still remains positive, even after treatment, the clinician should evaluate a different treatment for the disease or the possible presence of co-infections or other diseases [22]. qPCR is an effective tool to monitor treatment efficacy especially in those cases were repeated treatments are needed and to detect early possible relapses to avoid clinical disease.

As a preventive measure, annual screening is recommended for all dogs, especially those living in endemic areas.

\section{Conclusions}

In conclusion, due to the fact that qPCR allows quantification of parasite load in a precise manner, it could be an effective tool to confirm a diagnosis of canine leishmaniasis mostly (i) in those cases were serology is inconclusive, (ii) in cases where the dog has not yet seroconverted, (iii) for treatment monitoring. Dogs whose parasitemia range from medium to high or very high positive are sick or eventually will become sick of CanL.

\section{Acknowledgements}

Thanks to the veterinarians, dog owners and veterinary clinics that willingly provided the samples for this research. Publication of the CVBD6 thematic series has been sponsored by Bayer Animal Health $\mathrm{GmbH}$. This work was founded by the European commission (LUPA, GA-201370).

\section{Author details}

'Departament de Ciència Animal i dels Aliments, Facultat de Veterinària, Universitat Autònoma de Barcelona (UAB), 08193 Bellaterra, Barcelona, Spain. ${ }^{2}$ Servei Veterinari de Genètica Molecular, Departament de Ciència Animal i dels Aliments, Facultat de Veterinària, Universitat Autònoma de Barcelona, 08193 Bellaterra, Barcelona, Spain. ${ }^{3}$ Hospital Clínic Veterinari, Universitat Autònoma de Barcelona, 08193 Bellaterra, Barcelona, Spain.

\section{Authors' contributions}

VMD: Redacted the manuscript. VMD and JQO: collected samples, extracted DNA, performed $\mathrm{qPCR}$, performed phenotypic classification and edited the manuscript.

$X R, O F, A S B$ and LA: edited the manuscript. All authors read and approved the final manuscript.

\section{Competing interests}

The authors declare that they have no competing interests.

Received: 15 January 2011 Accepted: 13 April 2011

Published: 13 April 2011

\section{References}

1. Gramiccia M, Gradoni L: The current status of zoonotic leishmaniases and approaches to disease control. Int J Parasitol 2005, 35:1169-1180.

2. Ferroglio E, Maroli M, Gastaldo S, Mignone W, Rossi L: Canine leishmaniasis, Italy. Emerg Infect Dis 2005, 10:1618.

3. Petersen CA: Leishmaniasis, an Emerging Disease Found in Companion Animals in the United States. Top Companion Anim Med 2009, 24:182-188.

4. Martín-Sánchez J, Acedo C, Muñoz-Pérez M, Pesson B, Marchal O, MorillasMárquez F: Infection by Leishmania infantum in cats: Epidemiological study in Spain. Vet Parasitol 2007, 145:267-273.

5. Fernández-Bellon $\mathrm{H}$, Solano-Gallego L, Bardagí M, Alberola J, Ramis $\mathrm{A}$, Ferrer L: Immune response to Leishmania infantum in healthy horses in Spain. Vet Parasitol 2006, 135:181-185.

6. Sobrino R, Ferroglio E, Oleaga A, Romano A, Millan J, Revilla M, Arnal MC, Trisciuoglio A, Gortázar C: Characterization of widespread canine leishmaniasis among wild carnivores from Spain. Vet Parasitol 2008, 155:198-203.

7. Solano-Gallego L, Morell P, Arboix M, Alberola J, Ferrer L: Prevalence of Leishmania infantum infection in dogs living in an area of canine leishmaniasis endemicity using PCR on several tissues and serology. J Clin Microbiol 2001, 39:560-563.

8. Baneth $G$, Aroch I: Canine leishmaniasis: A diagnostic and clinical challenge. Vet J 2008, 175:14-15.

9. Gomes YM, Paiva Cavalcanti M, Lira RA, Abath FG, Alves LC: Diagnosis of canine visceral leishmaniasis: biotechnological advances. Vet J 2008, 175:45-52.

10. Cortés E, Sanz AJ, Vela C, Ranz Al: Leishmaniosis canina. Diagnóstico serológico de la leshmaniosis: análisis comparativo de ensayos inmunoenzimáticos e IFI. Inf Vet 2005, SEP:28-33.

11. Francino O, Altet L, Sanchez-Robert E, Rodriguez A, Solano-Gallego L, Alberola J, Ferrer L, Sanchez A, Roura X: Advantages of real-time PCR assay for diagnosis and monitoring of canine leishmaniosis. Vet Parasitol 2006, 137:214-221.

12. Stephan C, Wesseling S, Schink T, Jung K: Comparison of eight computer programs for receiver-operating characteristic analysis. Clin Chem 2003, 49(3):433-439.

13. Schoonjans F, Zalata A, Depuydt CE, Comhaire FH: MedCalc: a new computer program for medical statistics. Comput Methods Programs Biomed 1995, 48(3):257-262.

14. Paltrinieri S, Solano-Gallego L, Fondati A, Lubas G, Gradoni L, Castagnaro M, Crotti A, Maroli M, Oliva G, Roura X, Zatelli A, Zini E: Guidelines for diagnosis and clinical classification of leishmaniasis in dogs. J Am Vet Med Assoc 2010, 236:1184-1191. 
15. Strauss-Ayali D, Jaffe CL, Burshtain O, Gonen L, Baneth G: Polymerase chain reaction using noninvasively obtained samples, for the detection of Leishmania infantum DNA in dogs. J Infect Dis 2004, 189:1729-1733.

16. Moreno J, Alvar J: Canine leishmaniasis: epidemiological risk and the experimental model. Trends Parasitol 2002, 18:399-405.

17. Moreira MAB, Luvizotto MCR, Garcia JF, Corbett CEP, Laurenti MD: Comparison of parasitological, immunological and molecular methods for the diagnosis of leishmaniasis in dogs with different clinical signs. Vet Parasitol 2007, 145:245-252

18. Pena MT, Roura X, Davidson MG: Ocular and periocular manifestations of leishmaniasis in dogs: 105 cases (1993-1998). Vet Ophthalmol 2000, 3:35-41.

19. Koutinas AF, Polizopoulou ZS, Saridomichelakis MN, Argyriadis D, Fytianou A, Plevraki KG: Clinical considerations on canine visceral leishmaniasis in Greece: a retrospective study of 158 cases (1989-1996). J Am Anim Hosp Assoc 1999, 35:376-383.

20. Baneth G, Shaw SE: Chemotherapy of canine leishmaniosis. Vet Parasitol 2002, 106:315-324

21. Noli C, Auxilia ST: Treatment of canine Old World visceral leishmaniasis: a systematic review. Vet Dermatol 2005, 16:213-232.

22. Tabar MD, Francino O, Altet L, Sanchez A, Ferrer L, Roura X: PCR survey of vectorborne pathogens in dogs living in and around Barcelona, an area endemic for leishmaniasis. Vet Rec 2009, 164:112-116.

doi:10.1186/1756-3305-4-57

Cite this article as: Martínez et al.: Canine leishmaniasis: the key points for qPCR result interpretation. Parasites \& Vectors 2011 4:57.

\section{Submit your next manuscript to BioMed Central and take full advantage of:}

- Convenient online submission

- Thorough peer review

- No space constraints or color figure charges

- Immediate publication on acceptance

- Inclusion in PubMed, CAS, Scopus and Google Scholar

- Research which is freely available for redistribution

Submit your manuscript at www.biomedcentral.com/submit 